Musées, Patrimoine et Culture scientifiques et techniques

$128 \mid 2010$

mars - avril 2010

\title{
À propos du Patrimoine en questions de Françoise Choay
}

\section{André Desvallées}

\section{OpenEdition \\ Journals}

Édition électronique

URL : http://journals.openedition.org/ocim/163

DOI : 10.4000/ocim.163

ISSN : 2108-646X

Éditeur

OCIM

Édition imprimée

Date de publication : 1 mars 2010

Pagination : 36-39

ISSN : 0994-1908

\section{Référence électronique}

André Desvallées, «À propos du Patrimoine en questions de Françoise Choay », La Lettre de l'OCIM [En ligne], 128 | 2010, mis en ligne le 01 mars 2012, consulté le 30 avril 2019. URL : http:// journals.openedition.org/ocim/163; DOI : 10.4000/ocim.163 


\title{
Points de vue
}

\section{Lecture}

\section{À propos du Patrimoine en questions de Françoise Choay}

\author{
par André Desvallées, \\ Conservateur général honoraire du Patrimoine
}

Les publications sur le patrimoine sont foison. Et celles de Françoise Choay ne sont pas les moindres en intérêt, au nombre de trois ou quatre livres. Son Allégorie du patrimoine a sans doute été un des plus lus sur le sujet (peutêtre, par rapport à d'autres, parce que son éditeur avait une meilleure distribution). C'est pourquoi j'attendais le dernier (Patrimoine en questions. Paris : Seuil, 2009, 223 p.) avec impatience, qui se voulait une synthèse historique appuyée sur les meilleurs textes. Quelle n'est donc pas ma déception!

Ma déception tire sa source d'abord des imprécisions ou des lacunes qui proviennent d'une bibliographie lacunaire - j’allais écrire partisane ou sectaire. Nous nous plaignons des anglophones qui ne lisent pas les travaux des francophones. Ce n'est pas ici le cas. Nous nous plaignons aussi de ces universitaires qui ne lisent pas nos revues professionnelles alors que notre quotidien forme la matière de leurs études. C'est le cas ici. Expliqué, peut-être, mais aussi aggravé par le fait que Françoise Choay baigne dans un milieu particulier, celui des architectes et urbanistes, et celui des auteurs italiens.

Très positif a été pour nous le fait que François Choay nous ait révélé, par exemple, un Gustavo Giovannoni (1873-1947), architecte et théoricien de l'urbanisme et inventeur du concept (et des termes) de «patrimonio urbano », l'un des membres actifs de la Conférence d'Athènes de 1931 et sans doute inspirateur (indirect puisqu'il était décédé depuis 1947) de la Charte de Venise de 1964. Dans les bibliographies de Françoise Choay, on retrouve de nombreuses publications italiennes - et tant mieux pour combler les lacunes de notre savoir (toutefois généralement moins lacunaire que celui des Anglophones). Mais cet apport à notre culture vaut-il d'être compensé par d'énormes lacunes dans la culture de l'auteur lui-même?

En effet, s'agissant d'une anthologie (aux Éditions du Seuil, s'il vous plaît ! et avec une longue introduction prépubliée dans Esprit en novembre 2009) qui est sans doute destinée à être consultée par toute une génération d'élèves-architectes (comme l'a été L'Allégorie du patrimoine), comment peut-on se permettre de faire l'impasse totale sur les publications antérieures qui ont eu le même objectif (une sélection de textes touchant au patrimoine ou aux musées) ? Par exemple : en 1984, la première si je ne me trompe, de François Dagognet, Le musée sans fin ; en 1989, le gros volume de Bernard Deloche et Jean-Michel Leniaud, La Culture des sans-culottes (qui comprend notamment, in extenso, les Instructions sur la manière dinventorier et de conserver de Félix Vicq d'Azyr et dom Germain Poirier et les Quelques idées sur les arts de François Boissy d'Anglas) ; en 1993, Jean de Galard, Les Visiteurs du Louvre (dont l'abondance des textes excède largement le simple Louvre) ; en 2001, Dominique Poulot, Patrimoine et musées (qui n'est pas qu'une anthologie, mais contient de nombreux documents de toute époque classés par thèmes).

De même, si Françoise Choay a bien préfacé, en 1984, une traduction du Culte moderne des monuments d'Aloïs Riegl, introduit, en 1998, L'Urbanisme face aux villes anciennes de Giovannoni et édité, en 2000, les Mémoires du baron Haussmann, en 2002, le texte des conclusions de la Conférence d'Athènes sur la conservation artistique et historique des monuments, on peut se demander pourquoi elle fait un complet black-out sur les travaux comparables de ses collègues qui ont aussi été responsables de rééditions. Pour n’en citer que quelques unes parues depuis vingt ans : en 1989, à la fois la réédition, par Jean-Louis Déotte, des Considérations morales ainsi que des Lettres à Miranda de Quatremère de Quincy et l'édition critique, par Édouard Pommier, des mêmes Lettres à Miranda; celle, par le même, en 1992, des Réflexions sur le Muséum national de Jean-Baptiste-Pierre Le Brun ; ou, en 1991, la traduction annotée et l'introduction, par Marianne Charrière, des Réflexions sur l'Imitation des œuvres grecques de Johann J. Winckelmann.

Cet ouvrage cependant n'a pas que de mauvais côtés. Il a remis au jour des textes difficilement accessibles ou pas encore traduits : Suger, Bracciolini, Pie II, Raphaël, Aubin-Louis Millin (mais en oubliant de rappeler qu'il a aussi été, à partir du Directoire, avec André Barthélemy, 
conservateur des Antiques de la Bibliothèque nationale et qu'il eut, avec le même, un projet de musée universel) ; quelques réponses à l'enquête méconnue de 1794 ; la double lettre diatribe de Victor Hugo à l'adresse des démolisseurs ; des textes non traduits à ce jour de John Ruskin ; certains textes peu connus de Viollet-le-Duc ; des extraits de Giovannoni et des conclusions de la conférence d'Athènes auxquelles publications en français ou rééditions notre auteure avait déjà participé, comme je viens de le rappeler.

Néanmoins d'autres choix sont plus discutables : pourquoi en effet avoir choisi les voyages de Jacob Spon plutôt que ceux de Montaigne ou du Président Charles de Brosses ? Pourquoi avoir repris Vicq d'Azyr (et Poirier) avec de nombreux blancs, alors qu'il avait été repris intégralement dans Deloche et Leniaud ? Pourquoi avoir cité à peine une page et demie de Quatremère de Quincy, alors qu'il a écrit tellement de choses intéressantes (c'est trop ou trop peu, puisque, pour un cas semblable, aucune ligne de Boissy d'Anglas n'a été retenue ; en outre, si le passage cité est celui qui vise la création des musées, un autre, au moins aussi important, n’a pas été retenu, celui qui, dans les Lettres à Miranda, réclame le maintien in situ des vestiges romains,), mais aussi alors que deux de ses principaux textes ont été réédités ? Pourquoi n'avoir cité qu'à peine deux pages d'Aloïs Riegl : là aussi c'est trop ou trop peu puisque l'auteure avait préfacé l'intégralité du texte que l'on avait (enfin) traduit ?

Et puis, l'affaire s'aggrave vers la fin, avec Malraux d'abord, et ensuite avec les références internationales - et particulièrement l'UNESCO. Il n'est interdit à personne de rappeler que Malraux, avant d'être ministre, et même avant d'être romancier, a été un « mauvais garçon », pour avoir arraché des statues à un temple d'Angkor afin de les vendre pour éponger ses dettes. Il n'est pas interdit de rappeler qu'il était nationaliste et défendait (déjà !) l'exception française - mais dans un sens plus restrictif que ceux qui défendent en elle un universalisme s'opposant à l'individualisme et au communautarisme. Il n'est pas interdit de rappeler que sa culture n'était pas celle d'un professionnel des Monuments historiques ou de l'Inventaire - il en savait davantage sur les musées. Mais il n'est pas interdit non plus de se souvenir qu'on a appelé «loi Malranx » la loi n 62-903 du 4 août 1962, instituant les " secteurs sauvegardés », " complétant la législation sur la protection du patrimoine historique et esthétique de la France et tendant à faciliter la restauration immobilière » (1). Il n'est pas interdit non plus de rappeler qu'il était principalement un écrivain et qu'il avait, comme beaucoup, une conception de la culture privilégiant les Beaux-Arts et les chefsd'œuvre artistiques - encore que, dans ses livres, lorsqu'il a fait ses choix et ses comparaisons, les exemples qu'il a retenus ne répondaient pas toujours aux canons reconnus. Alors pourquoi n'avoir cité de lui que de courtes réponses à des questions de parlementaires ? Pourquoi n'avoir pas plutôt choisi des passages de ses livres sur l'art, ou retenu le discours de 1960 Pour sauver les monuments de Haute Égypte, beaucoup plus connu, il est vrai - mais qui aurait permis à Françoise Choay de remarquer que Malraux utilisait alors déjà le terme "patrimoine », comme nombre de ses collaborateurs, et que, lorsqu'il parlait de « Trésor », il ne pensait nullement à une valeur économique, mais bien à une richesse artistique ? Il n'est pas interdit enfin, puisqu'il était l'architecte préféré de Malraux, de rappeler que Le Corbusier avait été l'auteur d'un plan d'urbanisme (le « Plan Voisin ») qui envisageait la destruction des centres historiques des villes anciennes, comme Paris, afin de faire du neuf, mais cela n'oblige pas à prendre un ton méprisant à l'égard d'un architecte qui, s'il avait une vision non acceptable de l'urbanisme, n'en était pas moins un architecte génial.

Quant aux dispositions internationales de protection du patrimoine, pourquoi n'avoir retenu qu'à peine un article sur deux de la Charte dite de Venise de 1964 et de la Convention pour la protection du patrimoine mondial de l'UNESCO de 1972 ? L'une et l'autre ne sont pas d'une telle longueur qu'il ait fallu en celer la moitié. Et pourquoi n'avoir pas reproduit la toute récente Convention pour la sauvegarde du patrimoine culturel immatériel de 2003, qui confirme pourtant sans ambiguïté des tendances plus anciennes intégrant le culturel au naturel et ne mettant pas l'immatériel à part ?

J'ai comme une petite idée selon laquelle ces choix et ces amputations ne sont pas le fait du hasard - ni dûs au fait que l'espace était mesuré - car ils sont dans la logique de l'ensemble du propos.

Tout d'abord, le mépris qu'elle affiche pour les collègues d'une discipline très proche conduit malheureusement notre auteure à des erreurs parfois grossières. Par exemple il n'est pas peu banal de lui voir confondre (pp. 177 et 178) l'Office international des musées (OIM) - créé en 1926 et disparu du fait de la guerre - avec l'ICOM, créé seulement en 1946, même si leurs buts étaient comparables ${ }^{(2)}$. Autre exemple, ses références majoritairement italiennes l'empêchent trop souvent d'en connaître d'autres. C'est ainsi que, concernant son sujet (patrimoine et musées) tous les textes cités par Jean-Michel Leniaud, Édouard Pommier, Dominique Poulot et bien d'autres sont ignorés, qui auraient permis à notre auteure d'avoir une autre vision qu'esthétique, économique, et nationaliste a minima .

On peut en effet s'interroger sur les raisons qui ont poussé Françoise Choay à avancer la thèse, paradoxale dans le 
contexte, selon laquelle les valeurs du patrimoine mises en avant pour leur protection par les « antiquaires » de l'Ancien régime et de la Révolution française, auraient "une dimension économique dominante », alors que, en Angleterre, ils n'auraient qu' une valeur axiologique » (p. XXVII). Ce contresens est dû à une ignorance de l'étymologie et de l'évolution sémantique. Lorsqu'elle compare le sens de l'anglais « heritage » au français «patrimoine », en attribuant au terme anglais une valeur morale qu'elle refuse au terme français (p. XXVII), elle oublie que c'est justement pour échapper au sens notarial, attaché aux «biens », que le terme «patrimoine » avait été choisi à la fin du XVIIIe siècle et s'est diffusé progressivement au cours du siècle suivant, pour s'imposer définitivement dans les langues latines au XXe siècle. C'est d'ailleurs aussi sans doute pour la même raison que Giovannoni avait choisi, dès 1931, d'utiliser le terme «patrimonio » plutôt que l'expression « beni culturali ». Contrairement à ce qu'avance l'auteure, l'« axiologie » n'est pas le privilège des « antiquaires » anglais. Lorsqu'un « antiquaire » français, comme le susnommé Aubin-Louis Millin, présente ses Antiquités nationales ou Recueil de Monuments, il se lamente de ce que la vente de biens nationaux ait été " très funeste aux arts et aux sciences, en détruisant des produits du génie et des monuments historiques qu'il serait intéressant de conserver». Par « monuments nationaux » il entend "tous ceux qui peuvent retracer les grands événements de notre histoire devenue une des principales études des vrais citoyens ». Et lorsque le président Pétion le remercie de son travail, lors de la présentation de son travail à l'Assemblée nationale constituante, le 9 décembre 1790, il déclare : "[...] Sauver des ravages du temps qui consume tous ces antiques et précieux monuments du génie, c'est faire des conquêtes à l'empire de la raison. [...] L'Assemblée nationale se fera toujours un devoir de favoriser les progrès des sciences et des arts, tout ce qui peut illustrer les empires, et surtout conduire les hommes vers le bonheur; elle est trop convaincue que l'ignorance est la source de tous les manx». Il est toujours question des « sciences et des arts » et de leur " progrès ", jamais il n'est question de valeur économique. Il est question de "monuments du génie » « antiques et précieux », mais bien spécieuse est la lecture qui conduirait à donner à « précieux » le seul sens économique que peuvent leur donner les joailliers. Et pourtant, lorsque Vicq d'Azyr emploie le même terme en usant de l'expression «objets précieux », notre auteure ne peut s'empêcher d'en souligner «la dimension économique » (p. 88, note 4)!

D'après le Grand Robert, si le terme "précieux » est apparu au XIIe siècle dans son sens premier (du latin pretium = prix), dès le siècle suivant il s'était ajouté le second sens, d'ordre moral, sentimental, intellectuel... Et, lorsque La Fontaine nous parlera du « repos si précieux » nous aurons du mal à considérer que le repos des travailleurs ait, pour eux, une valeur marchande - même s'il en coûte à leur entreprise. Plus tard, lorsque Victor Hugo s'exclamera : "Il y a deux choses dans un édifice, son usage et sa beauté. Son usage appartient au propriétaire, sa beauté à tout le monde; c'est donc dépasser son droit que le détruire », qui pourrait prétendre qu'il mettait une valeur économique derrière la beauté, alors qu'il l'opposait aux droits de propriété et aux droits d'usage?

Certes, l'ambigüité remonte au trafic des reliques, elle est donc presque contemporaine de leur première apparition, presque aux origines de chaque religion. Mais, pour les croyants, les reliques n'ont pas de prix. La Joconde non plus, ni le château de Versailles. Si on leur donne un prix, c'est qu'on ne parle plus de la même chose. C'est qu'il ne s'agit plus de notre patrimoine, conservé pour l'étude ou la délectation, mais qu'il s'agit de marché. Il n’a fallu que deux siècles pour en arriver là. Mais, dans une époque où le l'économie et la finance dominent la politique et le savoir, où l'enseignement comme la santé se transforment en valeurs de marché, et où, en effet, on se met à vendre le patrimoine construit pour combler le déficit budgétaire, qui s'étonnerait de voir le concept de patrimoine se transformer aussi en marchandise ? De là à réinterpréter l'histoire à l'aune de la vision des banques et de la globalisation! Les cotes atteintes par les Monet, les Rubens, les Giacometti, les Picasso ou les Degas n'ont plus rien à voir avec le patrimoine culturel. Il s'agit de marché de l'art et c'est une toute autre histoire, même si les deux se croisent parfois à l'arrière des galeries ou dans les salles de ventes. Le patrimoine, c'est un état d'esprit, ce n'est pas un placement.

Il nous faut bien rappeler que lorsqu'on ignore Pommier on ignore aussi Puthod de Maison rouge, qu'il a remis au jour comme inventeur du terme «patrimoine national ». Et lorsqu'on ignore d'autres textes (3) sur l'origine du terme, on ne le voit apparaître qu'en 1931 et on lui donne comme seul sens celui de "monument d'art et d'histoire » (p.179). Mais c'est peut-être aussi ce qui empêche d'entrevoir que, dès la Révolution française, a vu le jour cette vision universaliste d'appropriation collective (qu'on retrouvera chez Renan) comme un phénomène non économique mais essentiellement culturel. On peut contester le concept de patrimoine pour des raisons politiques, en le replaçant dans le contexte colonial et dans une vision plus immatérielle de la culture, comme l'avait fait Stanislas Adotevi en $1971^{(4)}$; on peut constater que certains sont en train d'en dénaturer le sens en lui donnant hic et nunc ce sens économique qu'il n'avait pas chez les pères fondateurs il y a deux siècles.

Tout cela est bien dommage, dans un ouvrage qui va sans doute servir d'outil de travail à beaucoup ! 
laires en cour de construction : " s'il nous faut choisir entre la climatisation des personnes et celles des collections, nous choisissons les collections ".

(2) Si notre auteure s'était donnée la peine de regarder, par exemple, certaines publications de Pascal Ory, ou de Jacqueline Eidelman sur le Palais de la Découverte, ou, plus modestement, de moi-même dans ma présentation du texte de Louis Hautecœur sur l'Architecture et l'aménagement des musées, ou mon article sur l'origine du mot patrimoine, elle en aurait su davantage sur l'OIM, elle aurait découvert Euripide Foundoukidis, son secrétaire général, et sans doute évité d'imputer au seul Giovannoni, même si son rôle fut majeur, les évolutions conceptuelles et terminologiques des années 1930 et de l'Après-guerre.

\section{LOGICIEL DE GESTION DES COLLECTIONS MUSEALES}

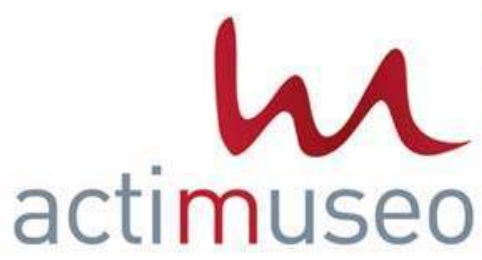

DÉJÀ +DE

150

MUSÉES

ÉQUIPÉS

Fiable et intuitif, le logiciel ActiMuséo couvre tous les aspects de la gestion des collections dans les musées: inventaire juridique et légal, documentation des œuvres, conservation et publication. Environnement PC, Mac ou Internet.

Inclus en standard dans la version 7 d'ActiMuséo:

- Registre d'inventaire,

- Base de récolement,

- Synchronisation des données,

- Export au format DMF.

VERSION MUSÉES: Beaux-arts, Art contemporain, Arts décoratifs, Archéologie, Ethnologie, Numismatique, Phaléristique, Manuscrits et Imprimés, Dépôt de fouilles archéologiques, Patrimoine architectural, Secteur sauvegardé, Sciences naturelles.

VERSION MUSÉUMS: Idem + Zoologie, Botanique, Pétrographie, Paléontologie, Minéralogie.
(3) Je pense en particulier à tous ceux que j’ai pu écrire depuis 1995 sur l'origine du mot... Mais il y en a bien d'autres.

(4) "L'internationalisation du concept de patrimoine de l'humanité n'est [...] pas seulement factice, mais dangereuse dans la mesure où l'on surimprime un ensemble de connaissances et de préjugés dont tous les critères sont les expressions de valeurs élaborées à partir de données esthétiques, morales, culturelles, bref de l'idéologie d'une caste dans une société dont les structures sont irréductibles à celles du Tiers Monde en général et de l'Afrique en particulier ». Adotevi, S. Le musée dans les systèmes éducatifs et culturels contemporains, Actes de la ge conférence générale de l'ICOM, Grenoble, 1971, p. 23. Repris dans : Négritude et négrologues. Paris : 10/18, 1972, pp. 283-284. et dans Vagues, tome 1, Mâcon : WW/Mnes, 1992, p. 127.

\section{SOLUTION DE VALORISATION DES} COLLECTIONS SUR INTERNET

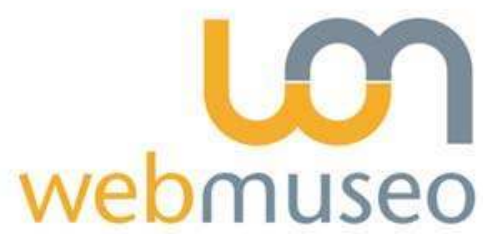

WebMuséo permet de répondre à de nombreux besoins des musées, en particulier la valorisation des collections sur Internet: publication des collections en ligne, expositions virtuelles, ... C'est aussi une solution simple et rapide pour la construction de portails culturels regroupant les collections de plusieurs musées, bibliothèques, médiathèques, ...

Privilégiant les technologies open source, WebMuséo s'adapte à tous les logiciels de gestion de collection.

Pour tout renseignement, contactez:

Xavier Maillot (xmaillot@aa-part.com)

Tél: 0970448812

Ou consultez le site www.aa-part.com 